\title{
PLASTIC DEFORMATION MECHANISMS \\ IN POLYIMIDE RESINS AND THEIR SEMI-INTERPENETRATING NETWORKS
}

\author{
Bor Z. Jang \\ Materials Science and Engineering \\ 201 Ross Hall \\ Auburn University, AL 36849 \\ Phone (205) 844-3324 \\ Fax (205) 844-3307 \\ NASA Co-workers: \\ Dr. Ruth H. Pater (ASEE/NASA Associate) \\ Dr. Mark Soucek \\ Dr. Jeff Hinkley
}

\section{PROJECT SUMMARY}

High-performance thermoset resins and composites are critical to the future growth of space, aircraft, and defense industries in the U.S.A. However, the processing-structureproperty relationships in these materials remain poorly understood. In the present ASEE/NASA Summer Research Program, the plastic deformation modes and toughening mechanisms in single-phase and multiphase thermoset resins were investigated. Both thermoplastic and thermoset polyimide resins and their interpenetrating networks (IPNs and semi-IPNs) were included in the present study. The fundamental tendency to undergo strain localization (crazing and shear banding) as opposed to a more diffuse (or homogeneous) deformation in these polymers were evaluated. Other possible toughening mechanisms in multiphase thermoset resins were also examined. The unique topological features of network chain configuration/conformation and the multiplicity of phase morphology in IPNs and semi-IPNs provide unprecedented opportunities for studying the toughening mechanisms in multiphase thermoset polymers and their fiber composites.

High performance semi-IPNs were prepared by combining a polyamic acid (a precursor to LaRC-TPI thermoplastic polyimide available from NASA LaRC or Mitsui Toatsu, or NR150B2 from Du Pont) with PMR-15 molding powder through solution mixing. PMR-15, one of the NASA-developed thermoset polyimide resins, possesses a good thermal stability but very poor toughness. The concept of high performance interpenetrating networks (IPNs) combines an easy-to-process but brittle thermoset with a tough but difficult-to-process thermoplastic polymer. This combination has led to the development of numerous high temperature semi-IPNs that could be processed like a thermoset and possess good toughness like a thermoplastic. Our first achievement in this Summer Research Program was to successfully cast thin films of PMR-15 from a solution state, which was considered to be extremely difficult if not impossible. For the first time, thin films of NR-150B2 thermoplastic polyimide and several series of NR-150B2- and LaRC-TPI-based semi-IPNs were obtained by solvent casting. 


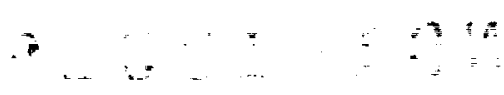

Thin films of various compositions were cut into rectangular specimens for in-situ polarizing light microscopic observation of crack-tip deformation mechanisms with a miniature tensioning device attached onto the microscope stage. Mechanisms near, ahead of, and in the wake of a crack tip were examined in-situ at various stages of loading. LaRC TPI was found to exhibit combined shear yielding and crazing under plane-stress loading conditions. In response to tensile loading, the single-edge-notched TPI specimen appeared to show some initial diffuse shear yielding, which was quickly turned into a more localized combined shear-banding/crazing mode. Crazes appeared to grow at a slightly faster rate than shear bands and therefore always led the way in development of the deformation zone ahead of a growing crack tip. Final failure of the specimen involves the propagation of the crack through the center or along the edge of the deformation zone. Contrarily, NR-150B2 exhibited diffuse shear yielding; no shear banding or crazing was found near the crack tip.

Adding a small amount of thermoplastic component, either NR-150B2 or LaRC TPI, was found to significantly improve the fracture toughness of PMR-15 thermoset PI. PMR15 films also showed diffuse shear yielding with a small deformation zone. This can be understood on the basis of a limited extensibility of a highly cross-linked network. Numerical calculations were performed to confirm this low value of network chain draw ratio. The dimensions of the deformation zone ahead of the crack tip was increased as a higher weight fraction of the thermoplastic component was added. On the other end of the fracture toughness spectrum, the deformation zone size of a thermoplastic matrix was found to scale down with an increasing amount of thermoset PMR-15. Deformation also became more diffuse with a higher PMR-15 content in LaRC TPI. Fracture toughness variations can be correlated with deformation mode changes in these semi-IPNs.

In the near future, various semi-IPNs with controlled phase morphologies and network structures will be fabricated by manipulating the backbone structure, component fractions and phase separation kinetics. The effects of these parameters on the mechanical behavior of these semi-IPNs will be examined. Several other approaches will also be taken to determine the dominant plastic deformation mechanisms in a semi-IPN resin or composite. 